\title{
Costs, Complications, and Reoperations Associated With Primary Arthroscopic Rotator Cuff Repair With or Without Acromioplasty and/or Biceps Tenodesis
}

\author{
Kunal Varshneya, B.S., Marc R. Safran, M.D., Seth L. Sherman, M.D., and \\ Geoffrey D. Abrams, M.D.
}

\begin{abstract}
Purpose: To evaluate the reoperations, complications, and costs up to 5 years following arthroscopic rotator cuff repair (RCR) alone, with acromioplasty (acro), with biceps tenodesis (BT), or with both acro and BT. Methods: We queried the MarketScan database to identify patients who underwent RCR from 2007 to 2016 . Patients were stratified into groups based on concomitant procedures (acro and/or BT) performed on the same day as index RCR. Reoperations, complications, and costs were followed for 5 years post-index procedure. Patients without laterality codes were excluded. A multivariate logistic regression analysis was used to control for confounding factors. Results: This study identified 147,838 patients (mean age, 53.1 years; standard deviation, 8.3 years) who underwent primary RCR. Patients were stratified into 4 groups: (1) RCR only, (2) RCR + acro, (3) RCR + BT, and (4) RCR + acro + BT. Patients in the RCR only group experienced the highest rate of unadjusted overall postoperative complications $(17.2 \%)$ versus the other groups (RCR + acro $16.4 \%, \mathrm{RCR}+\mathrm{BT} 15.1 \%, \mathrm{RCR}+$ acro $+\mathrm{BT} 16.2 \%, P<.0161)$. The RCR only group also experienced a significantly greater number of reoperations on the ipsilateral shoulder $(P<.0001)$, whereas the RCR + acro + BT had the highest costs at all timepoints. In the regression analysis, there was no significant differences between complications and reoperations between any groups. After adjusting for covariates, the performance of a BT with an RCR and acromioplasty led to increased costs (odds ratio, 1.47, 1.37-1.59, $P<.001$ ). Conclusions: Concomitant biceps tenodesis does lead to higher total healthcare costs, both in the shorter and longer terms. When adjusting for confounding factors, the performance of concomitant biceps tenodesis with rotator cuff repair does not lead to a difference in postoperative complication rate or risk for revision surgery. Level of Evidence: Level IV, economic analysis.
\end{abstract}

From the Department of Orthopaedic Surgery, Stanford University Medical Center, Palo Alto, California, U.S.A.

The authors report the following potential conflicts of interest or sources of funding: M.R.S.: American Journal of Sports Medicine: editorial or governing board; Biomimedica: stock or stock options and unpaid consultant; DJ Orthopaedics: IP royalties; International Society for Hip Arthroscopy: board or committee member; International Society of Arthroscopy, Knee Surgery, and Orthopaedic Sports Medicine: board or committee member, JISAKOS: editorial or governing board; Journal of Hip Preservation Surgery: editorial or governing board; Medacta: paid consultant, paid presenter, or speaker; Saunders/Mosby-Elsevier: publishing royalties, financial, or material support; Smith $\theta$ Nephew: IP royalties, paid presenter or speaker, research support; Stryker: IP royalties; Wolters Kluwer Health-Lippincott Williams $\theta$ Wilkins: publishing royalties, financial or material support. S.L.S.: ACL Study Group: board or committee member; American Journal of Orthopedics: editorial or governing board; American Orthopaedic Society for Sports Medicine: board or committee member; Arthrex, Inc: paid consultant and research support; Arthroscopy: editorial or governing board; Arthroscopy Association of North America: board or committee member; Ceterix Orthopaedics: paid consultant; CONMED Linvatec: paid consultant; Flexion Therapeutics: paid consultant; GLG Consulting: paid consultant; International Cartilage Regeneration $\theta$ Joint Preservation Society: board or committee member; International Society of Arthroscopy, Knee Surgery, and Orthopaedic Sports Medicine: board or committee member; JRF Ortho: paid consultant; Moximed: paid consultant; Olympus: paid consultant; Vericel: paid consultant. G.D.A.: AAOS: board or committee member; American Orthopaedic Society for Sports Medicine: board or committee member; Arthrex, Inc: other financial or material support; Cytonics Inc.: paid consultant and stock or stock options; Fidia Pharma: paid consultant; International Society of Arthroscopy, Knee Surgery, and Orthopaedic Sports Medicine: board or committee member; RubiconMD: paid consultant; Sideline Sports Doc: paid consultant; Stryker: other financial or material support; TeachAIDS: unpaid consultant. Full ICMJE author disclosure forms are available for this article online, as supplementary material.

Received October 20, 2019; accepted May 11, 2020.

Address correspondence to Geoffrey Abrams, Orthopaedic Surgery, Stanford University Medical Center, 450 Broadway St, Redwood City, CA 94063 U.S.A. E-mail: geoffa@stanford.edu

(C) 2020 THE AUTHORS. Published by Elsevier Inc. on behalf of the Arthroscopy Association of North America. This is an open access article under the CC BY-NC-ND license (http://creativecommons.org/licenses/by-nc-nd/4.0/). 2666-061X/191252

https://doi.org/10.1016/j.asmr.2020.05.010 
$\mathbf{R}$ otator cuff tears (RCTs) are a common orthopaedic pathology that account for upwards of 4.5 million annual visits to physicians. ${ }^{1}$ The incidence of RCTs increases with age, with an estimated $10 \%$ of individuals above the age of 60 and up to $50 \%$ of individuals older than age 80 having full-thickness tears of the RCT. ${ }^{2}$ Rotator cuff repairs (RCRs) can be performed using both arthroscopic and open techniques, with both having comparable clinical outcomes and complication rates. $^{3-6}$

Procedures such as acromioplasty (acro) and biceps tenodesis (BT) are frequently performed in conjunction with a RCR. ${ }^{7}$ Acromioplasties are used when the acromion is considered the culprit of the RCT and/or there may be clinically significant impingement. ${ }^{8-10}$ The clinical benefit of these additional procedures, however, remains unclear because previous reports have identified no functional benefit or reduction of revision risk with the concomitant use of an acro in RCR. ${ }^{11-14}$

Tenotomy or tenodesis is often favored when long head of the biceps tendon pathology is noted during surgery, with all techniques having similar clinical outcomes. ${ }^{15-19}$ Lesions of the long head of the biceps tendon (LHBT) are also often associated with RCTs and may be managed nonoperatively, with a tenotomy, or a BT. ${ }^{20-23}$ Tenotomy and tenodesis have been shown to have similar outcomes, but tenotomy has been shown to have an increased rate of cramping and deformity. ${ }^{21}$ Biceps tenodesis techniques differ by approach (open vs arthroscopic), location (proximal, suprapectoral, subpectoral), and fixation type (soft tissue to soft tissue vs soft tissue to bone). ${ }^{22-26}$

Although functional and clinical outcomes following BT with or without RCR repair are favorable, ${ }^{24,27-29}$ concerns remain about the cost effectiveness of BT over the long-term. Arthroscopic BT has been shown to have increased costs when compared with open procedures, $^{30}$ but few data exist regarding the cost-effectiveness of arthroscopic BT alone or in combination with acro when examining total procedural and postoperative costs.

The purpose of this study was to evaluate the reoperations, complications, and costs up to 5 years following RCR alone, with acro, with BT, or with both acro and BT. We hypothesize that the concomitant use of a BT will lead to increase costs in the short-term, however through long-term follow-up, become financially equivalent with RCR by itself.

\section{Methods}

\section{Data Source}

This study obtained a sample of the MarketScan Commercial Claims and Encounters database (Truven Health Analytics, Ann Arbor, MI) from January 1, 2007, to December 31, 2016. This database is a collection of commercial inpatient, outpatient, and pharmaceutical claims of more than 75 million employees, retirees, and dependents representing a substantial portion of the US population covered by employer-sponsored insurance. MarketScan contains 53 million patient inpatient records, 40 million with employer-sponsored insurance, 3.7 million with Medicare Part B, and 6.8 million on Medicaid for a total of more than 28 billion patient records. The data are updated quarterly, with all new records becoming available within 15 months of service and $91 \%$ of claims available within 5 months. Because of MarketScan's sourcing from large employers, its data boast superior longitudinal tracking of patients. Truven Health Analytics MarketScan data sets are publicly available to researchers for a fee per year of data. The MarketScan database contains International Classification of Diseases, 9th Revision, Clinical Modification (ICD-9-CM) and 10th revision, Clinical Modification, Current Procedural Terminology (CPT), Diagnosis Related Group codes, as well as National Drug Codes.

\section{Inclusion Criteria}

This study identified 147,838 patients who underwent primary RCR surgery (CPT 29827) between 2007 and 2016. Other procedures performed on the same day of index surgery were identified by the following CPT codes: Acromioplasty (CPT 29826) and Biceps Tenodesis (CPT 23430 or 29828). Only patients with confirmed laterality were included in this study. Individual demographic information and comorbidity status including history of diabetes, hyperlipidemia, arterial hypertension, obesity, osteoporosis, and tobacco use of each patient were gathered. Laterality of surgery was also noted (Fig 1).

\section{Outcomes}

The primary outcome measure of the study was 5-year costs after primary RCR, and we compared this for RCR alone versus with BT, acro, or both BT and acro. A secondary goal was to determine postoperative complication and revision surgery rates. The presence of a postoperative complication was defined as a complication occurring within 90 days of the index RCR surgery. These included infection (998.5-998.59, 730.0-730.91, 996.66, 996.67) capsulitis (726.0, 719.51), hematoma (998.1, 998.11, 998.12, 998.13), shoulder dislocation (831.00, 831.09, 718.31, 718.21), nerve injury (955.0-955.9, 907.4), or wound complications (998.3, 998.31, 998.32, 998.81, 998.83, 998.4, 101.40, 101.60, 101.80, deep vein thrombosis [451.0-453.9], and pulmonary embolism [415.1-415.19]). Thromboembolism was defined as presence of either a pulmonary embolism or deep vein thrombosis. A revision surgery was defined by the presence of the aforementioned RCR CPT codes for after the index surgery date and on the ipsilateral shoulder. This 


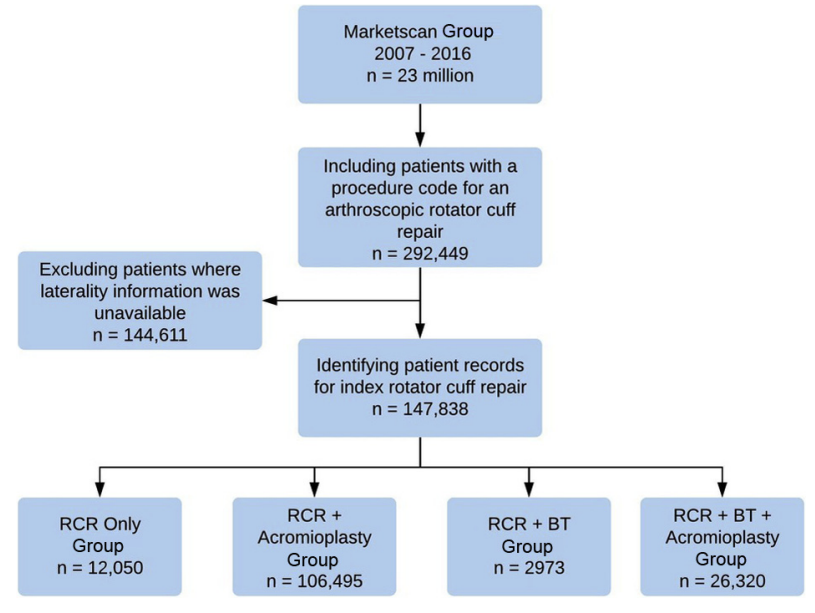

Fig 1. Group diagram

was measured at 90 days, 6 months, 1 year, and 2 years following index surgery. Health care utilization data were also collected. Immediate payments (same day of surgery) as well as payments through 9 months, 2 years, and 5 years were included. Procedure specific payments were also analyzed. This was defined as a payment on the same day of surgery directly attributed to the procedure (by the presence of an RCR CPT code in the service reimbursed).

\section{Patient Groups and Statistical Analysis}

Patients were stratified into surgical groups based on the concomitant procedures performed on the same day of index surgery: RCR only, RCR + acro, RCR + BT, or $\mathrm{RCR}+$ acro $+\mathrm{BT}$. If patient records lacked the codes for acro and BT, there were classified as RCR only. Followup time was measured, and no significant differences were noted between the procedures. This allowed us to conclude that loss to follow-up was similar among the groups, and comparative analyses of the long-term outcomes would be fair. To minimize the effect of potential confounding on the direct comparison of patients undergoing the different RCR procedures, a multivariate multiple logistic regression analysis was conducted. This methodology controlled for baseline covariates such as age, sex, and Charlson Comorbidity Index. Two sample $t$ tests, rank-sum and $\chi 2$ tests were used to assess significant differences in unadjusted demographic data, postoperative complications and reoperation rates, and payments among the groups. An alpha value of 0.05 was set as significant.

\section{Results}

\section{Patient Group}

A total of 147,838 patients met the inclusion criteria of this study, with each group being mutually exclusive (Table 1). RCR-only patients were significantly younger than the other 3 groups (49.6 years vs 53.2 vs 52.4 vs $54.3, P<.0001)$. RCR only $(41.1 \%)$ and RCR + acro patients $(44.2 \%)$ has a similar percentage of females, whereas patients who had a BT procedure had significantly fewer female proportions (RCR + BT: $31.1 \%$, $\mathrm{RCR}+\mathrm{BT}+$ acro $34.2 \%)(P<.0001)$. Rotator cuff repair $+\mathrm{BT}+$ acro patients had the highest rates of hypertension $(47.4 \%, P<.0001)$ whereas RCR + acro patients had the highest rates of diabetes $(17.3 \%$, $P<.001)$ and osteoporosis $(6.7 \%, P<.0001)$.

\section{Complications and Revision Surgery}

In the unadjusted data, there is an increase in postoperative complications in the non-BT groups. Patients in the RCR-only group experienced the highest rate of postoperative complications $(17.2 \%)$ compared with the other surgical approaches (RCR + acro $16.4 \%$, $\mathrm{RCR}+\mathrm{BT} 15.1 \%, \mathrm{RCR}+$ acro + BT $16.2 \%, P<.0161)$. Patients undergoing RCR + BT had the highest rates of wound complication $(0.27 \%, P=.0016)$, whereas patients undergoing RCR + acro had the highest rates of capsulitis $(14.5 \%, P<.0001)$. Rates of thromboembolism, nerve injury, infection, and hematoma were similar among surgical approaches (Table 2). In multivariate regression, the performance of a BT does not lead to higher complications rates $(P>.05)$.

Table 1. Demographics and Baseline Comorbidities

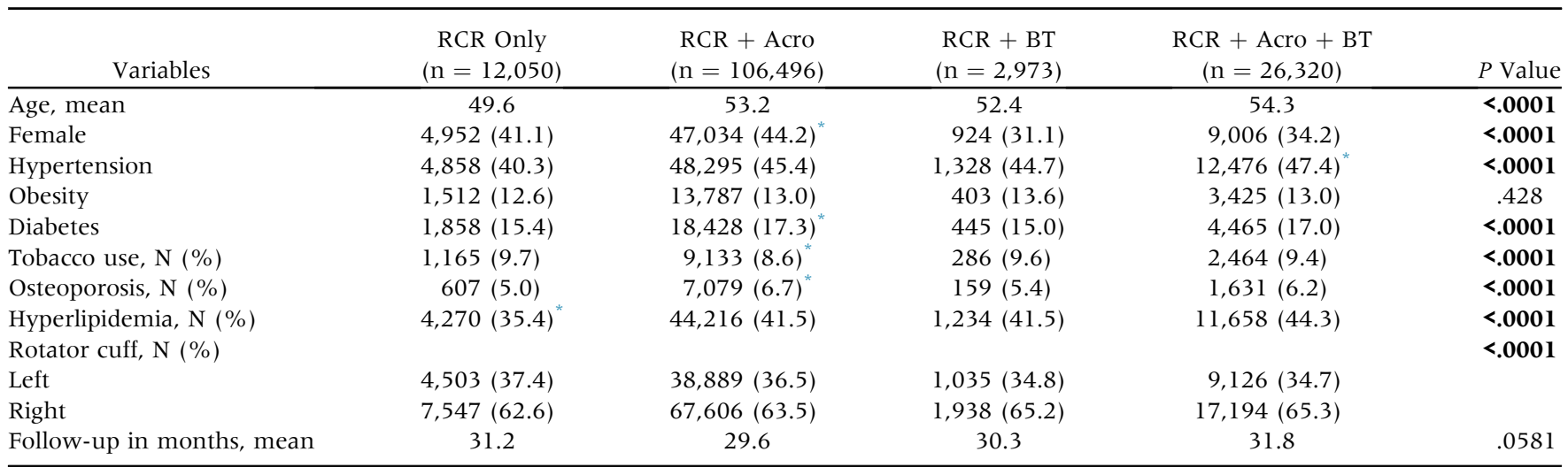

NOTE. Boldface indicates statistical significance $(P<.05)$.

*Driver of significance. 
Table 2. Postoperative Complications

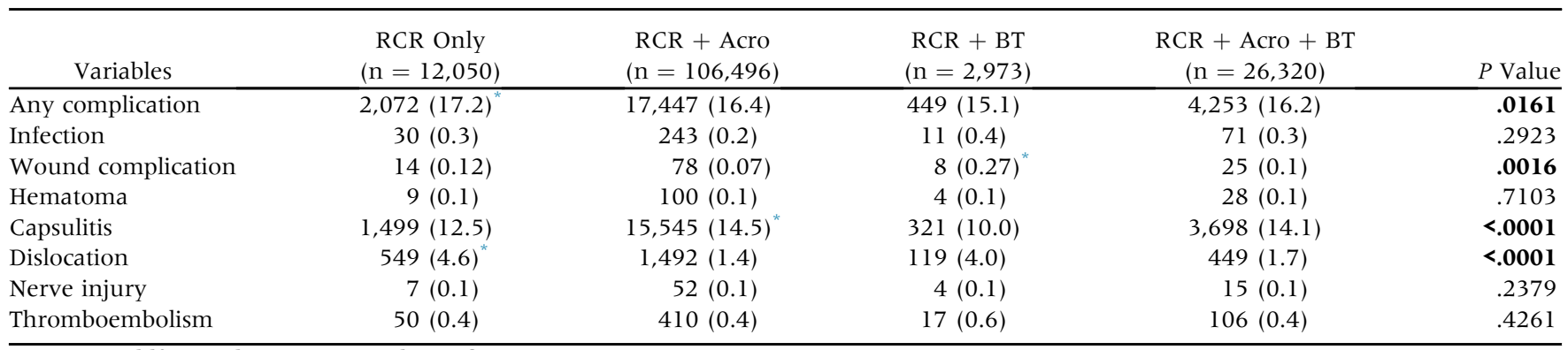

NOTE. Boldface indicates statistical significance $(P<.05)$.

*Driver of significance.

In the unadjusted data, the RCR-only group had an increased reoperation rate at all time points following surgery $(P<.0001)$ (Table 3$)$. Within 90 days, the RCR-only patients had a $1.4 \%$ revision surgery rate compared with $1.3 \%$ in the BT groups. This difference remained through 2 years of follow-up, with RCR-only patients having a $2.9 \%$ revision rate, whereas RCR + BT had a $2.8 \%$ and RCR + BT + acro had a $2.4 \%$ revision rate $(P<.0001)$ (Table 3$)$. In multivariate regression analysis, RCR + BT and RCR + BT + acro did not lead to higher rates of revision surgery $(P>.05)$ (Table 4$)$.

\section{Healthcare Utilization}

Rotator cuff repair with acromioplasty and biceps tenodesis had the highest immediate costs (Table 5). Procedure specific payments ranged from RCR only being the least expensive $(\$ 3,364)$ to RCR + acro + BT costing $\$ 6,787$. When comparing different BT techniques, open BT had a median cost of $\$ 5,406$, whereas median arthroscopic BT cost was $\$ 5,803$. Although the difference between the total health care utilization decreased over time, RCR + BT + acro remained the most expensive through 5 years of follow-up $(\$ 28,434, P<.0001)$ (Table 6). This was confirmed in a regression analysis as well, as the performance of a BT alongside RCR + Acro led to increased odds of greater than average costs (odds ratio 1.47, 1.37-1.59, $P<.001$ ). (Table 4 ).

\section{Discussion}

After adjusting for confounding variables, the performance of a BT did lead to higher costs both immediately and through 5 years of follow-up but had similar rates of overall complications and revision surgeries.

This study used a large national billing and claims dataset to conduct a comparative analysis of patients undergoing RCR with and without acromioplasties and BT from 2007 through 2016. and discusses the costeffectiveness related to the concomitant use of BT during RCR. The performance of BT led to an increase in immediate costs. This is to be expected, as the concomitant use of BT not only increases the operative time, but also the technical complexity of the surgery. Interestingly, the elevation of BT related costs remained in long-term follow-up at 5 years. Rotator cuff repair resource utilization is a concern because data indicate payments associated with them increased $144 \%$ from 2001 through 2009, whereas hospital payments only increased $85 \% .^{31}$ Significant efforts needs to be made to optimize patient selection for concomitant RCR procedures to mitigate the increasing burden on the health care system.

The cost-effectiveness of RCRs have been of significant interest in the orthopedic community. Adla et al. ${ }^{32}$ argued that arthroscopic RCR (ARCR) may be less costeffective than open cuff repair because the arthroscopic portion led to higher payments but no difference in clinical metrics such as inpatient time, amount of analgesia, number of outpatient visits, or physiotherapy use. This finding was corroborated Kose et al., ${ }^{33}$ finding similar clinical results between ARCR and mini-open RCR but higher payments associated with ARCR.

Table 3. Revision Surgery

\begin{tabular}{|c|c|c|c|c|c|}
\hline Variables & $\begin{array}{c}\text { RCR Only } \\
(\mathrm{n}=12,018)\end{array}$ & $\begin{array}{l}\text { RCR + Acro } \\
(\mathrm{n}=106,191)\end{array}$ & $\begin{array}{l}\mathrm{RCR}+\mathrm{BT} \\
(\mathrm{n}=2,968)\end{array}$ & $\begin{array}{c}\mathrm{RCR}+\text { Acro }+\mathrm{BT} \\
(\mathrm{n}=26,320)\end{array}$ & $P$ Value \\
\hline Within 30 days & $151(1.3)^{*}$ & $838(0.8)$ & $37(1.3)$ & $326(1.2)$ & $<.0001$ \\
\hline Within 90 days & $163(1.4)^{*}$ & $965(0.9)$ & $39(1.3)$ & $346(1.3)$ & $<.0001$ \\
\hline Within 6 months & $225(1.9)^{*}$ & $1,338(1.2)$ & $57(1.9)$ & 447 (1.7) & $<.0001$ \\
\hline Within 1 year & $296(2.5)^{*}$ & $1,819(1.7)$ & $74(2.5)$ & $568(2.2)$ & $<.0001$ \\
\hline Within 2 year & $346(2.9)^{*}$ & $2,126(2.0)$ & $83(2.8)$ & $623(2.4)$ & $<.0001$ \\
\hline
\end{tabular}

NOTE. Boldface indicates statistical significance $(P<.05)$.

*Driver of significance in post hoc analysis. 
Table 4. Multivariate Regression to Determine Independent Association of Approach Type with Outcomes

\begin{tabular}{|c|c|c|c|c|c|c|c|c|c|}
\hline \multirow[b]{2}{*}{ Variables } & \multicolumn{3}{|c|}{ Any Complication } & \multicolumn{3}{|c|}{ Any Reoperation } & \multicolumn{3}{|c|}{ Greater than Average Costs } \\
\hline & OR & $95 \% \mathrm{CI}$ & $P$ & OR & $95 \% \mathrm{CI}$ & $P$ & OR & $95 \% \mathrm{CI}$ & $P$ \\
\hline Rotator cuff repair only & 1.03 & 0.97-1.09 & .3181 & 1 & $0.93-1.07$ & .991 & 0.31 & $0.29-0.32$ & $<.0001$ \\
\hline $\mathrm{RCR}+$ biceps tenodesis & 0.91 & $0.82-1.0$ & .0925 & 1.1 & $0.96-1.28$ & .1503 & 0.68 & $0.63-0.73$ & $<.0001$ \\
\hline $\begin{array}{l}\text { Rotator cuff repair }+ \text { biceps } \\
\text { tenodesis }+ \text { acromioplasty }\end{array}$ & 1 & $0.97-1.0$ & .7992 & 1 & $0.95-1.05$ & .988 & 1.47 & $1.37-1.59$ & $<.0001$ \\
\hline
\end{tabular}

NOTE. Boldface indicates statistical significance $(P<.05)$.

$\mathrm{CI}$, confidence interval; OR, odds ratio.

Churchill et al. ${ }^{34}$ built upon these data and identified decreased operating room times and significantly reduced costs with mini-open compared to ARCR. Although the financial consequences of acromioplasties have also been investigated, and in cases of shoulder impingement syndrome, 1 study showed no clinical benefit but significantly increased costs, a more recent study has shown better long-term outcomes of isolated acromioplasty in comparison to PT alone for impingement syndrome. ${ }^{35,36}$

This study also identified differing rates of adverse events between the procedures. The postoperative complication rate was the highest in the RCR only group, which also had a mean age significantly younger than the other procedure groups. This is to be expected because younger patients are typically more active and therefore have higher chances of shoulder dislocations and subsequent pathology. ${ }^{37-40}$ Patients undergoing BT had higher rates of wound complications than the other patients. This is consistent with Nho et al.' $\mathrm{s}^{41}$ study that identified a rate of $0.28 \%$ of patients developing wound infections necessitating irrigate and debridement. This can be explained by the increased operative time needed in arthroscopic BT or the necessity for an additional incision in an open $\mathrm{BT} .^{42-44}$

The surgical management of RCTs typically includes subacromial decompression in conjunction with the repair of the damaged tendons. ${ }^{45}$ The role of subacromial impingement of the rotator cuff was initially described by Neer at al. ${ }^{46}$ in 1972 , with a recommendation of partial resection of the acromion to reduce friction and damage of the tendons in contact. Although outcomes following RCR with concomitant acromioplasty are favorable, ${ }^{11,47,48}$ a concern remains regarding the effectiveness of acromioplasty. Interestingly, in this study, $89.8 \%$ of the patients undergoing RCR had a concomitant acromioplasty, indicating its prevalence in use. Several randomized controlled trials have described little to no difference in the clinical and functional outcomes in the performance of RCR with or without acromioplasties. ${ }^{1,49,50}$ However, 1 investigation was able to identify a reduction in revision risk following RCR with acromioplasty compared with RCR alone and indicate that acromioplasty may be indicated in rotator cuff disease with a type III acromion. ${ }^{11,45}$

Lesions of the biceps tendon are often identified during RCR, but are also associated with superior labrum anterior posterior tears and glenohumeral arthritis. ${ }^{51-53}$ Pathology of LHBT is often associated with anterior shoulder pain, with surgical intervention being indicated in situations of biceps instability, tenosynovitis, tendinosis, or acute or degenerative tearing. ${ }^{54}$ Surgical options include biceps tenotomy or tenodesis; however, considerable debate remains regarding the optimal surgical strategy. ${ }^{19,30,55,56}$ Both strategies provide strong clinical outcomes, however, tenotomy has less associated surgical morbidity and tenodesis may provide better maintenance of supination strength. ${ }^{56}$ In the context of rotator cuff repairs, biceps tenotomy or tenodesis may further relieve pain and increase shoulder function. ${ }^{27}$ The severity of RCT and LHBT has been suggested to be directly proportional, with $16 \%$ to $75 \%$ of RCT having some concomitant LHBT pathology. ${ }^{53}$ Previous studies have determined that patients who undergo RCR with any concomitant biceps tendon surgery (biceps tenodesis

Table 5. Costs Outcomes

\begin{tabular}{|c|c|c|c|c|c|}
\hline Immediate payments & $\$ 10,079$ & $\$ 11,545$ & $\$ 13,284$ & $\$ 15,157$ & $<.0001$ \\
\hline 9 months & $\$ 15,766$ & $\$ 17,277$ & $\$ 19,217$ & $\$ 21,236$ & $<.0001$ \\
\hline 2 years & $\$ 20,085$ & $\$ 21,600$ & $\$ 23,298$ & $\$ 25,313$ & $<.0001$ \\
\hline 5 years & $\$ 24,033$ & $\$ 25,644$ & $\$ 25,914$ & $\$ 28,434$ & $<.0001$ \\
\hline
\end{tabular}

NOTE. Boldface indicates statistical significance $(P<.05)$.

*Driver of significance in post hoc analysis. 
Table 6. Procedural Costs

\begin{tabular}{lccc}
\hline \multicolumn{1}{c}{ Variables } & Median & $\begin{array}{c}25 \text { th } \\
\text { Percentile }\end{array}$ & $\begin{array}{c}\text { 75th } \\
\text { Percentile }\end{array}$ \\
\hline RCR only & $\$ 3,364$ & $\$ 1,957$ & $\$ 5,462$ \\
RCR and acromioplasty & $\$ 4,867$ & $\$ 3,258$ & $\$ 7,122$ \\
$\begin{array}{l}\text { RCR and biceps tenodesis } \\
\text { (open) }\end{array}$ & $\$ 5,406$ & $\$ 3,680$ & $\$ 8,176$ \\
$\begin{array}{l}\text { RCR and biceps tenodesis } \\
\text { (scope) }\end{array}$ & $\$ 5,803$ & $\$ 3,844$ & $\$ 8,612$ \\
$\begin{array}{l}\text { RCR and acromioplasty } \\
\text { and any biceps tenodesis }\end{array}$ & $\$ 6,787$ & $\$ 4,609$ & $\$ 10,191$ \\
\hline
\end{tabular}

or biceps tenotomy) present with lower baseline functional status (indicating more severe pathology) but have greater improvements in patient-reported outcomes when compared with those who underwent isolated RCR. ${ }^{27}$ Meraner et al. ${ }^{57}$ analyzed 53 patients undergoing RCR with concomitant biceps tenodesis or tenotomy procedures and found no difference in patient satisfaction or functional outcome scores. Zhang et al. further corroborated these findings in a study of 151 patients, but also noted there was no increased rate of Popeye deformity in the biceps tenodesis group. Erickson et al. conducted the first large-scale study investigating the risk for revision surgery following RCR and biceps tenodesis. In a study of 29,827 patients derived from an administrative database, they identified an increase rate of reoperation at 6 months and 1 year following concomitant BT and RCR, when compared with isolated RCR. ${ }^{29}$ This is in contrast to the data of this study, which after adjusting for confounding variables, found no increased risk for reoperation following BT. One reason for the discrepancy may be in the nature of the administrative coding; in this study, confirmed laterality was required to consider a follow-up procedure as a revision; a criterion the previous study did not use that may have led to underestimate of revision risk in this study or overestimation of risk in theirs.

\section{Limitations}

As with all large database studies, interpretations of this study are limited by the retrospective and administrative nature of these data. Because patients, procedures, and clinical outcomes were queried using ICD-9-CM and CPT codes, it is not possible to assess the underlying validity of the collected records. Our analysis is limited by the accuracy and completeness of the codes. There may be underestimations regarding postoperative complications if the administrators did not appropriately code them. Using a large dataset such as MarketScan provides for powerful analyses but has an inherent level of selection bias and lacks clinical details, specifically shoulder functional status, radiographic findings, detailed physical examinations, clinical outcomes, and more detailed long-term follow-up. The lack of data regarding the size and chronicity of RCT particularly affected our ability to stratify the group by severity of disease. In addition, the location of where the biceps tenodesis was performed was also unavailable. Furthermore, the data provided from MarketScan do not provide us with a specific indication for surgery. Preoperative morbidity may have served as a confound in that patients with increased morbidity were selected to not receive a biceps tenodesis. Also, patients frequently switch health plans, so it is possible that our longer term costs and complication data are less robust versus early follow-up data. We conducted a comparative cost analysis of the use of concomitant procedures in RCR and may provide useful insight into to the complications, costs, and revision rate of RCRs with and without concomitant procedures.

\section{Conclusions}

Concomitant biceps tenodesis does lead to higher total health care costs, both in the short and longer terms. When adjusting for confounding factors, the performance of concomitant biceps tenodesis with RCR does not lead to a difference in postoperative complication rate or risk for revision surgery.

\section{References}

1. Aleem AW, Brophy RH. Outcomes of rotator cuff surgery: What does the evidence tell us? Clin Sports Med 2012;31: 665-674.

2. Reilly P, Macleod I, Macfarlane R, Windley J, Emery RJ. Dead men and radiologists don't lie: A review of cadaveric and radiological studies of rotator cuff tear prevalence. Ann R Coll Surg Engl 2006;88:116-121.

3. Shan L, Fu D, Chen K, Cai Z, Li G. All-arthroscopic versus mini-open repair of small to large sized rotator cuff tears: A meta-analysis of clinical outcomes. PLoS One 2014;9: e94421.

4. Ji X, Bi C, Wang F, Wang Q. Arthroscopic versus miniopen rotator cuff repair: An up-to-date meta-analysis of randomized controlled trials. Arthroscopy 2015;31: 118-124.

5. Morse K, Davis AD, Afra R, Kaye EK, Schepsis A, Voloshin I. Arthroscopic versus mini-open rotator cuff repair: A comprehensive review and meta-analysis. Am J Sports Med 2008;36:1824-1828.

6. Osti L, Papalia R, Paganelli M, Denaro E, Maffulli N. Arthroscopic vs mini-open rotator cuff repair. A quality of life impairment study. Int Orthop 2010;34:389-394.

7. Jensen AR, Cha PS, Devana SK, et al. Evaluation of the trends, concomitant procedures, and complications with open and arthroscopic rotator cuff repairs in the medicare population. Orthop J Sports Med 2017;5: 2325967117731310.

8. Bigliani LU, Ticker JB, Flatow EL, Soslowsky LJ, Mow VC. The relationship of acromial architecture to rotator cuff disease. Clin Sports Med 1991;10:823-838. 
9. Flatow EL, Soslowsky LJ, Ticker JB, et al. Excursion of the rotator cuff under the acromion. Patterns of subacromial contact. Am J Sports Med 1994;22:779-788.

10. Nicholson GP, Goodman DA, Flatow EL, Bigliani LU. The acromion: Morphologic condition and age-related changes. A study of 420 scapulas. J Shoulder Elbow Surg 1996;5:1-11.

11. Abrams GD, Gupta AK, Hussey KE, et al. Arthroscopic repair of full-thickness rotator cuff tears with and without acromioplasty: Randomized prospective trial with 2-year follow-up. Am J Sports Med 2014;42:1296-1303.

12. Song L, Miao L, Zhang P, Wang WL. Does concomitant acromioplasty facilitate arthroscopic repair of fullthickness rotator cuff tears? A meta-analysis with trial sequential analysis of randomized controlled trials. Springerplus 2016;5:685.

13. Jarvis DL, Waterman BR, Verma NN. Is acromioplasty ever indicated during rotator cuff repair? Arthroscopy 2019;35:1639-1640

14. Jaeger M, Berndt T, Rühmann O, Lerch S. Patients with impingement syndrome with and without rotator cuff tears do well 20 years after arthroscopic subacromial decompression. Arthroscopy 2016;32:409-415.

15. Werner BC, Evans CL, Holzgrefe RE, et al. Arthroscopic suprapectoral and open subpectoral biceps tenodesis: A comparison of minimum 2-year clinical outcomes. Am J Sports Med 2014;42:2583-2590.

16. Werner BC, Brockmeier SF, Gwathmey FW. Trends in long head biceps tenodesis. Am J Sports Med 2015;43: 570-578.

17. Lee HI, Shon MS, Koh KH, Lim TK, Heo J, Yoo JC. Clinical and radiologic results of arthroscopic biceps tenodesis with suture anchor in the setting of rotator cuff tear. J Shoulder Elbow Surg 2014;23:e53-e60.

18. Koh KH, Ahn JH, Kim SM, Yoo JC. Treatment of biceps tendon lesions in the setting of rotator cuff tears: Prospective cohort study of tenotomy versus tenodesis. Am J Sports Med 2010;38:1584-1590.

19. Shank JR, Singleton SB, Braun S, et al. A comparison of forearm supination and elbow flexion strength in patients with long head of the biceps tenotomy or tenodesis. Arthroscop 2011;27:9-16.

20. Ahmad CS, ElAttrache NS. Arthroscopic biceps tenodesis. Orthop Clin North Am 2003;34:499-506.

21. Hassan S, Patel V. Biceps tenodesis versus biceps tenotomy for biceps tendinitis without rotator cuff tears. J Clin Orthop Trauma 2019;10:248-256.

22. Hong $\mathrm{CK}$, Yeh ML, Chang $\mathrm{CH}$, et al. Comparison of changes in shoulder functions between biceps tenotomy and tenodesis in an animal model. Asia Pac J Sports Med Arthrosc Rehabil Technol 2019;15:17-22.

23. Hufeland M, Wicke S, Verde PE, Krauspe R, Patzer T. Biceps tenodesis versus tenotomy in isolated LHB lesions: A prospective randomized clinical trial. Arch Orthop Trauma Surg 2019;139:961-970.

24. Green JM, Getelman MH, Snyder SJ, Burns JP. Allarthroscopic suprapectoral versus open subpectoral tenodesis of the long head of the biceps brachii without the use of interference screws. Arthroscopy 2017;33: 19-25.
25. Gombera MM, Kahlenberg CA, Nair R, Saltzman MD, Terry MA. All-arthroscopic suprapectoral versus open subpectoral tenodesis of the long head of the biceps brachii. Am J Sports Med 2015;43:1077-1083.

26. Brady PC, Narbona P, Adams CR, et al. Arthroscopic proximal biceps tenodesis at the articular margin: Evaluation of outcomes, complications, and revision rate. Arthroscopy 2015;31:470-476.

27. Watson ST, Robbins CB, Bedi A, Carpenter JE, Gagnier JJ, Miller BS. Comparison of outcomes 1 year after rotator cuff repair with and without concomitant biceps surgery. Arthroscopy 2017;33:1928-1936.

28. Xiao MAG. Increased re-operation rates among patients undergoing shoulder arthroscopy with concomitant biceps tenodesis. JSES Open Access 2019;3:344-349.

29. Erickson BJ, Basques BA, Griffin JW, et al. The effect of concomitant biceps tenodesis on reoperation rates after rotator cuff repair: A review of a large private-payer database from 2007 to 2014. Arthroscopy 2017;33: 1301-1307.

30. Forsythe B, Agarwalla A, Puzzitiello RN, Mascarenhas R, Werner BC. Rates and risk factors for revision open and arthroscopic proximal biceps tenodesis. Orthop J Sports Med 2019;7:2325967118825473.

31. Iyengar JJ, Samagh SP, Schairer W, Singh G, Valone FH, Feeley BT. Current trends in rotator cuff repair: Surgical technique, setting, and cost. Arthroscopy 2014;30:284-288.

32. Adla DN, Rowsell M, Pandey R. Cost-effectiveness of open versus arthroscopic rotator cuff repair. J Shoulder Elbow Surg 2010;19:258-261.

33. Köse KC, Tezen E, Cebesoy O, et al. Mini-open versus allarthroscopic rotator cuff repair: Comparison of the operative costs and the clinical outcomes. Adv Ther 2008;25: 249-259.

34. Churchill RS, Ghorai JK. Total cost and operating room time comparison of rotator cuff repair techniques at low, intermediate, and high volume centers: Mini-open versus all-arthroscopic. J Shoulder Elbow Surg 2010;19:716-721.

35. Ketola S, Lehtinen J, Arnala I, et al. Does arthroscopic acromioplasty provide any additional value in the treatment of shoulder impingement syndrome? A two-year randomised controlled trial. J Bone Joint Surg Br 2009;91: 1326-1334.

36. Farfaras S, Sernert N, Rostgard Christensen L, Hallström EK, Kartus JT. Subacromial decompression yields a better clinical outcome than therapy alone: A prospective randomized study of patients with a minimum 10-year follow-up. Am J Sports Med 2018;46: 1397-1407.

37. Mather RC, Koenig L, Acevedo D, et al. The societal and economic value of rotator cuff repair. J Bone Joint Surg Am 2013;95:1993-2000.

38. Hattrup SJ. Rotator cuff repair: Relevance of patient age. J Shoulder Elbow Surg 1995:4:95-100.

39. Tempelhof S, Rupp S, Seil R. Age-related prevalence of rotator cuff tears in asymptomatic shoulders. J Shoulder Elbow Surg 1999;8:296-299.

40. Tashjian RZ. Epidemiology, natural history, and indications for treatment of rotator cuff tears. Clin Sports Med 2012;31:589-604. 
41. Nho SJ, Reiff SN, Verma NN, Slabaugh MA, Mazzocca AD, Romeo AA. Complications associated with subpectoral biceps tenodesis: Low rates of incidence following surgery. J Shoulder Elbow Surg 2010;19:764-768.

42. Duchman KR, DeMik DE, Uribe B, Wolf BR, Bollier M. Open versus arthroscopic biceps tenodesis: A comparison of functional outcomes. Iowa Orthop J 2016;36:79-87.

43. Yi Y, Lee JM, Kwon SH, Kim JW. Arthroscopic proximal versus open subpectoral biceps tenodesis with arthroscopic repair of small- or medium-sized rotator cuff tears. Knee Surg Sports Traumatol Arthrosc 2016;24:3772-3778.

44. Gilmer BB, DeMers AM, Guerrero D, Reid JB, Lubowitz JH, Guttmann D. Arthroscopic versus open comparison of long head of biceps tendon visualization and pathology in patients requiring tenodesis. Arthroscopy 2015;31:29-34.

45. MacDonald P, McRae S, Leiter J, Mascarenhas R, Lapner P. Arthroscopic rotator cuff repair with and without acromioplasty in the treatment of full-thickness rotator cuff tears: A multicenter, randomized controlled trial. J Bone Joint Surg Am 2011;93:1953-1960.

46. Neer CS. Anterior acromioplasty for the chronic impingement syndrome in the shoulder: A preliminary report. J Bone Joint Surg Am 1972;54:41-50.

47. Blevins FT, Warren RF, Cavo C, et al. Arthroscopic assisted rotator cuff repair: Results using a mini-open deltoid splitting approach. Arthroscopy 1996;12:50-59.

48. Romeo AA, Hang DW, Bach BR, Shott S. Repair of full thickness rotator cuff tears. Gender, age, and other factors affecting outcome. Clin Orthop Relat Res 1999:243-255.

49. Gartsman GM, O'connor DP. Arthroscopic rotator cuff repair with and without arthroscopic subacromial decompression: A prospective, randomized study of oneyear outcomes. J Shoulder Elbow Surg 2004;13:424-426.

50. Milano G, Grasso A, Salvatore M, Zarelli D, Deriu L, Fabbriciani C. Arthroscopic rotator cuff repair with and without subacromial decompression: A prospective randomized study. Arthroscopy 2007;23:81-88.

51. Ahrens PM, Boileau P. The long head of biceps and associated tendinopathy. J Bone Joint Surg Br 2007;89: $1001-1009$.

52. Biz C, Vinanti GB, Rossato A, Arnaldi E, Aldegheri R. Prospective study of three surgical procedures for long head biceps tendinopathy associated with rotator cuff tears. Muscles Ligaments Tendons J 2012:2:133-136.

53. Chen $\mathrm{CH}, \mathrm{Hsu} \mathrm{KY}$, Chen WJ, Shih $\mathrm{CH}$. Incidence and severity of biceps long head tendon lesion in patients with complete rotator cuff tears. J Trauma 2005;58:1189-1193.

54. Barber A, Field LD, Ryu R. Biceps tendon and superior labrum injuries: decision-marking. J Bone Joint Surg Am 2007;89:1844-1855.

55. Schrock JB, Kraeutler MJ, Bravman JT. Comparison of clinical failure rates after 2 techniques of subpectoral mini-open biceps tenodesis: Sequence and suture passage technique matter. Orthop J Sports Med 2017;5:2325967117729356.

56. Friedman JL, FitzPatrick JL, Rylander LS, Bennett C, Vidal AF, McCarty EC. Biceps tenotomy versus tenodesis in active patients younger than 55 years: Is there a difference in strength and outcomes? Orthop J Sports Med 2015;3:2325967115570848.

57. Meraner D, Sternberg C, Vega J, Hahne J, Kleine M, Leuzinger J. Arthroscopic tenodesis versus tenotomy of the long head of biceps tendon in simultaneous rotator cuff repair. Arch Orthop Trauma Surg 2016;136:101-106. 